\title{
miRNA-214 suppresses oxidative stress in diabetic nephropathy via the ROS/Akt/mTOR signaling pathway and uncoupling protein 2
}

\author{
SHUFANG YANG ${ }^{1}$, XIAOQIANG FEI ${ }^{1}$, YU LU $^{1}$, BANGKUI XU ${ }^{1}$, YONGMEI MA ${ }^{1}$ and HUI WAN ${ }^{2}$ \\ ${ }^{1}$ Department of Endocrinology, Taizhou People's Hospital, Fifth People's Hospital of Nantong University, Taizhou, \\ Jiangsu 225300; ${ }^{2}$ Department of Endocrinology, Wuxi Second People's Hospital, Wuxi, Jiangsu 214000, P.R. China
}

Received March 28, 2018; Accepted September 6, 2018

DOI: $10.3892 /$ etm.2019.7359

\begin{abstract}
In the present study, the function of microRNA (miR)-214 on diabetic nephropathy (DN) and diabetes of proximal tubular cells was investigated. Reverse transcriptionquantitative polymerase chain reaction was used measure the expression of miR-214 in rats with DN and ELISA was performed to measure oxidative stress and ROS levels. Results indicated that miR-214 expression in the peripheral blood was significantly decreased in rats with DN. The in vitro model of DN indicated that miR-214 upregulation significantly decreased oxidative stress and reactive oxygen species (ROS) levels, but significantly increased uncoupling protein 2 (UCP2), phosphorylated (p)-Akt and p-mammalian target of rapamycin (mTOR) protein expression levels. The administration of genipin, a UCP2 inhibitor, significantly attenuated the effects of miR-214 upregulation on oxidative stress in the in vitro $\mathrm{DN}$ model by regulating ROS, Akt and mTOR protein expression levels. Notably, Akt inhibitor suppressed p-Akt protein expression and attenuated the effects of miR-214 upregulation on oxidative stress in the in vitro $\mathrm{DN}$ model. Collectively, these data suggest that miR-214 regulates diabetes through a ROS/Akt/mTOR signaling pathway by UCP2 in proximal tubular cells.
\end{abstract}

\section{Introduction}

Diabetic nephropathy (DN) is the most common complication induced by diabetes, which is one of the major reasons for renal failure (1). Its morbidity is increased gradually year by year, making it a global public health challenge that severely damages human health (1). It is estimated by the World Trade

Correspondence to: Dr Shufang Yang, Department of Endocrinology, Taizhou People's Hospital, Fifth People's Hospital of Nantong University, 366 Taihu Road, Taizhou, Jiangsu 225300, P.R. China

E-mail: yopptghhq9618@126.com

Key words: microRNA-214, diabetic nephropathy, UCP2, reactive oxygen species, Akt, mammalian target of rapamycin
Organization that the number of diabetics in the world will rise to 0.37 billion by 2025 , and $30 \%$ of these cases will develop DN (2). The pathological characteristics of DN primarily include glomerular hypertrophy and hyperplasia, basilar membrane thickening, and increased extracellular matrix. DN gradually develops into glomerular sclerosis, interstitial fibrosis as well as loss of function (3). Eventually, DN results in chronic renal failure, which reduces quality of life and endangers the patient's life (3). There are multiple pathogeneses responsible for DN. However, these are complicated processes that have not been completely illuminated yet (4).

Uncoupling protein (UCP) is a type of proton channel localized on the inner mitochondrial membrane (5). UCP belongs to the mitochondrial carrier protein superfamily (5). It reduces the electrochemical gradient on both sides of membrane, decreases ATP production and leads to reduced production of reactive oxygen species (ROS) in mitochondria (5). These findings suggest the protective effects of UCP on tissues. $\mathrm{UCP} 2$ is extensively distributed in multiple sites, including the liver, brain, kidney, gland membrane, muscle, white adipose tissue, retinal endothelial cells and pericytes (6). Previous findings have indicated that UCP2 mRNA expression can be upregulated in the presence of elevated intracellular ROS levels (6). Furthermore, overexpression of UCP2 analogue or antioxidase can reduce the level of ROS, which may prevent the occurrence of complications associated with diabetes (7).

MicroRNAs (miRs) are endogenous, non-coding, small RNA molecules that are 20-24 nucleotides long (8). miRs are also extensively distributed molecules that regulate gene expression (8). It is currently believed that miRs serve important roles during the growth and development of organisms. miRs can regulate genes at a post-transcription level. Thus, they are involved in almost all physiological and pathological processes in the body (9). These processes include cell proliferation, differentiation, apoptosis and immune inflammation (9). Previous research in recent years has suggested that miRs serve important roles in the genesis and development of DN through gene regulation, suggesting miRs may be associated with the genesis and development of DN $(9,10)$. Although the understanding towards the biological characteristics of miRs and their roles in DN has improved $(9,10)$, further studies are required. Investigating the association of DN with miRs will provide a novel viewpoint for 
understanding the genesis and development of DN. In addition, this may also provide biomarkers for the early diagnosis of DN, and novel targets for treatment and intervention. The current study aimed to address the function of miR-214 on DN and diabetes in proximal tubular cells.

\section{Materials and methods}

Animals. The present study was approved by the Taizhou People's Hospital Ethics Committee (Taizhou, China). Male Sprague Dawley rats (5-6 weeks old, 200-220 g) had unrestricted access to food and water and were maintained in a controlled environment (temperature, $22-23^{\circ} \mathrm{C}$; humidity, 55-60\%; and a 12-h light (7:00)/dark (19:00) cycle). All rats were randomly assigned into a sham $(n=6)$ or a DN model $(n=6)$ group. Animals in the sham group were injected once with normal saline. The DN model rats were injected once with $75 \mathrm{mg} / \mathrm{kg}$ streptozocin (Sigma-Aldrich; Merck KGaA, Darmstadt, Germany) (11). At 12 weeks after the induction of diabetes, mice were anesthetized with $35 \mathrm{mg} / \mathrm{kg}$ of pentobarbital sodium (intraperitoneal, Sigma-Aldrich; Merck KGaA) and sacrificed using decollation.

Hematoxylin and eosin staining. Kidney tissue samples were fixed with $4 \%$ paraformaldehyde for $24 \mathrm{~h}$ at room temperature and processed using paraffin at $55-60^{\circ} \mathrm{C}$ for $5-10 \mathrm{~min}$. Paraffin-embedded tissue sections $(10 \mu \mathrm{m})$ were deparaffinized using xylene for $60 \mathrm{~min}$ at $60^{\circ} \mathrm{C}$, hydrated using ethyl alcohol at room temperature for $30 \mathrm{~min}$ and stained with $0.5 \%$ hematoxylin for $10 \mathrm{~min}$ at room temperature, washed with water for $5 \mathrm{~min}$ and $0.5 \%$ eosin for $1 \mathrm{~min}$ at room temperature. Sections were observed using a light microscope (magnification, x200).

Reverse transcription-quantitative polymerase chain reaction $(R T-q P C R)$. Total RNA was extracted from cells or serum following the induction of the model using an RNA extraction kit (Invitrogen; Thermo Fisher Scientific, Inc., Waltham, MA, USA). cDNA was reverse transcribed using a Q-script kit (Quanta Biosciences, Gaithersburg, MD, USA) and a TaqMan miR reverse transcription kit (Applied Biosystems; Thermo Fisher Scientific, Inc.) at $37^{\circ} \mathrm{C}$ for $1 \mathrm{~h}$ followed by $82^{\circ} \mathrm{C}$ for 10 sec. qPCR was performed using an ABI Prism 7500 Sequence Detection System (Perkin-Elmer Inc., Waltham, MA, USA) and a standard SYBR Green PCR kit (Toyobo Life Science, Osaka, Japan). The temperature protocol was as follows: Initial denaturation at $95^{\circ} \mathrm{C}$ for $5 \mathrm{~min}$; followed by 40 cycles of denaturation at $95^{\circ} \mathrm{C}$ for $30 \mathrm{sec}$, annealing at $60^{\circ} \mathrm{C}$ for $30 \mathrm{sec}$ and elongation at $72^{\circ} \mathrm{C}$ for $30 \mathrm{sec}$. Primer sequences were as follows: miR-214, forward, 5'-AGCATAATACAGCAG GCACAGAC-3' and reverse, 5'-AAAGGTTGTTCTCCACTC TCTCAC-3'; U6, forward, 5'-ATTGGAACGATACAGAGA AGATT-3' and reverse, 5'-GGAACGCTTCACGAATTTG-3'. Results were analyzed using the $2^{-\Delta \Delta \mathrm{Cq}}$ method (12).

Gene expression profiling. Total RNA was hybridized to Affymetrix HG-U133 Plus 2.0 GeneChip arrays (Affymetrix; Thermo Fisher Scientific, Inc.). Data were analyzed using the database for annotation, visualization and integrated discovery and Qiagen Ingenuity Pathway Analysis (Qiagen, Inc., Valencia, CA, USA).
Cell culture and transfection. Human HK-2 renal proximal tubular epithelial cells were cultured in Dulbecco's modified Eagle's medium (DMEM) supplemented with $10 \%$ fetal bovine serum (Thermo Fisher Scientific, Inc.) and 1\% penicillin/streptomycin. miR-214-5p (100 ng; 5'-GGCCTGGCT GGACAGAGTTG-3') and negative control (100 ng; 5'-CCC CCCCCCCCCC-3') were purchased from Sangon Biotech Co., Ltd. (Shanghai, China). miR-214 mimics and mimic-negative control were transfected with Lipofectamine 2000 (Thermo Fisher Scientific, Inc.). Following 48 h of transfection, cells were cultured in DMEM supplemented with $35 \mathrm{mM}$ glucose for $24 \mathrm{~h}$ for further analysis. Cells were then treated with $2 \mathrm{nM}$ MK 2206 dihydrochloride (Akt inhibitor; MedChemExpress, Shanghai, China) or UCP2 inhibitor (genipin; $50 \mu \mathrm{M}$; MedChemExpress, Monmouth Junction, NJ, USA) for $44 \mathrm{~h}$ and cultured in DMEM supplemented with $35 \mathrm{mM}$ of glucose for $24 \mathrm{~h}$ for further analysis.

ELISA kits. Following the induction of DN, supernatants of cells were collected using centrifugation at 1,000 $\mathrm{x} \mathrm{g}$ for $5 \mathrm{~min}$ at $4^{\circ} \mathrm{C}$ and $10-50 \mu \mathrm{l}$ of supernatant were used to analyze the levels of oxidative stress [superoxide dismutase (SOD), A001-1-1; malondialdehyde (MDA), A003-1; glutathione (GSH), A006-2; GSH-peroxidase (PX), A005)] and ROS (E004) using ELISA kits (Nanjing Jiancheng Bioengineering Institute, Nanjing, China). The fluorescence intensity was measured using a spectrofluorometer (Fluorostar, BMG Labtech $\mathrm{GmbH}$, Ortenberg, Germany) at $450 \mathrm{~nm}$. Cells were stained with dichloro-dihydro-fluorescein diacetate $(10 \mu \mathrm{M})$ for $20 \mathrm{~min}$ at $37^{\circ} \mathrm{C}$ and ROS levels were observed using a Zeiss Axioplan 2 (magnification, x200; Zeiss AG, Oberkochen, Germany).

Western blot analysis. Cells were lysed using radioimmunoprecipitation assay lysis buffer (Beyotime Institute of Biotechnology, Haimen, China) for $15 \mathrm{~min}$ at $4^{\circ} \mathrm{C}$ and the protein content was then determined using a BCA Protein Assay (Thermo Fisher Scientific, Inc.). Equal amounts $(50 \mu \mathrm{g})$ of protein were separated by SDS-PAGE $(10 \%$ gels) and blotted onto polyvinylidene difluoride membranes (Bio-Rad Laboratories, Inc., Hercules, CA, USA). Blots were blocked at room temperature with $5 \%$ non-fat dry milk in Tris-buffered Saline with Tween 20 (TBST) for $1 \mathrm{~h}$ and incubated with UCP2 (89326; 1:2,000), phosphorylated (p)-Akt $(4060 ; 1: 2,000)$, p-mammalian target of rapamycin (mTOR; 5536; 1:2,000) and GAPDH (5174; 1:5,000; Cell Signaling Technology, Inc., Danvers, MA, USA) overnight at $4^{\circ} \mathrm{C}$. Membranes were exposed to anti-rabbit horseradish peroxidase-conjugated secondary antibody (7074; 1:5,000, Cell Signaling Technology, Inc.) for $1 \mathrm{~h}$ at $37^{\circ} \mathrm{C}$, following several washes with TBST. Membranes were detected using ECL Plus reagents (GE Healthcare, Chicago, IL, USA) and quantified using the Molecular Imager ChemiDoc XRS System and Quantity One 1-D analysis software (Bio-Rad Laboratories, Inc.).

Luciferase activity. The 3'-untranslated region (UTR) of UCP2 was cloned into luciferase reporter vector (CmiT000001MT06; GeneCopoeia, Inc., Rockville, MD, USA). The 3'UTR of UCP2 and miR-214 mimics (100 ng) were co-transfected 

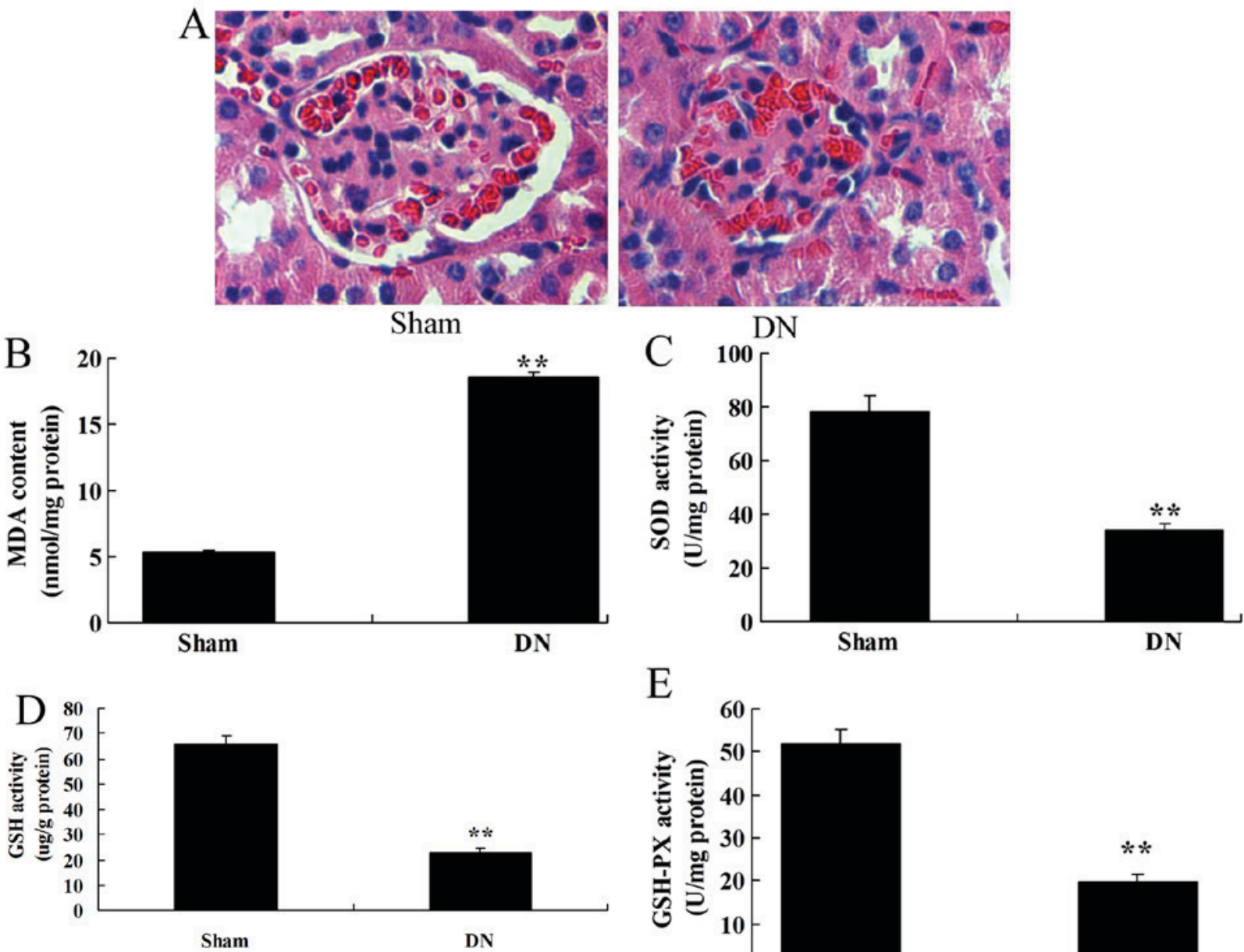

$\mathrm{E}$

$\mathrm{F}$
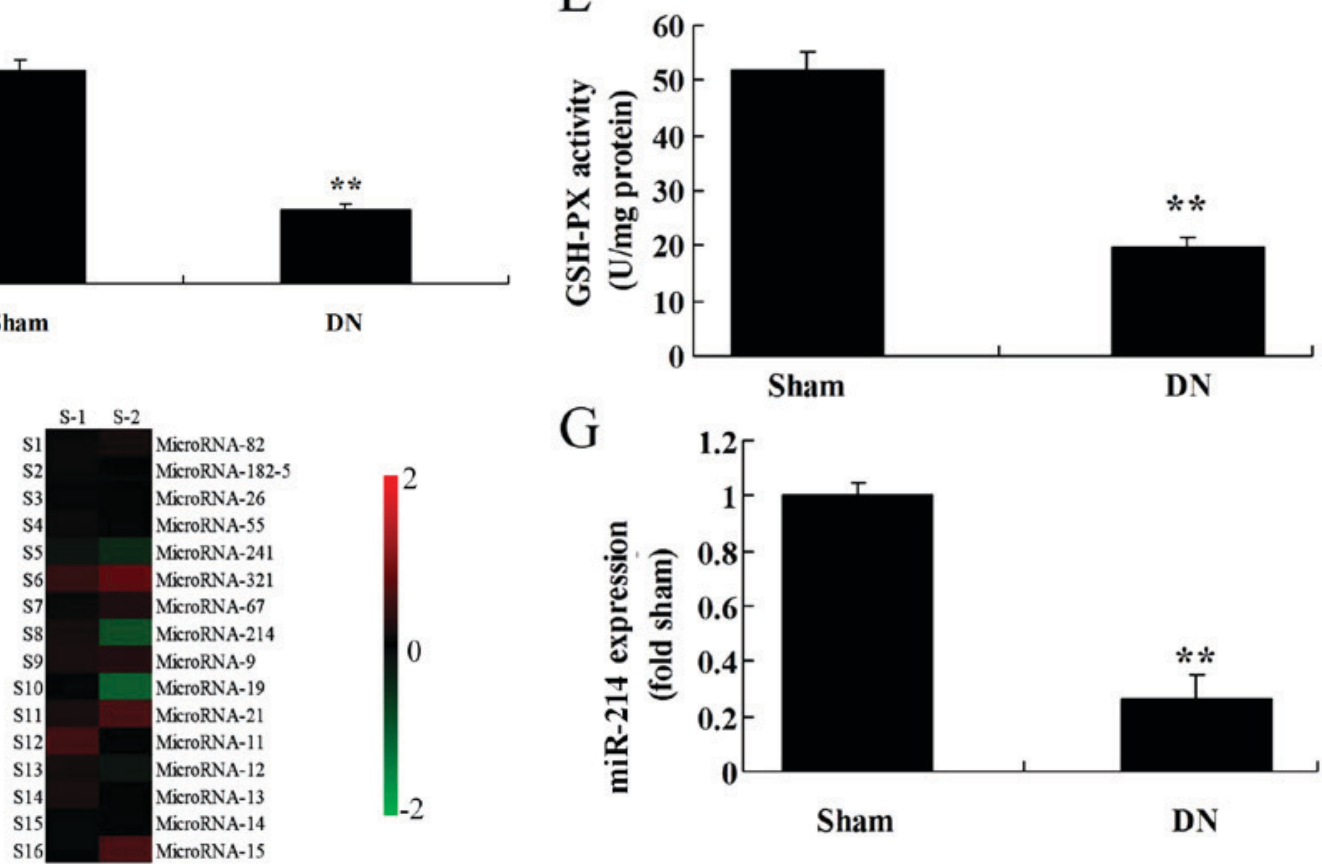

G

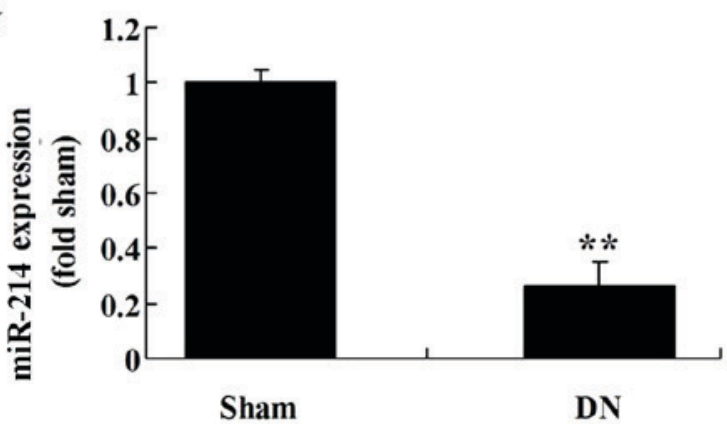

Figure 1. miR-214 expression in rats with DN. Hematoxylin and eosin staining for (A) glomerulus (magnification, x200). ELISA was used to determine (B) MDA, (C) SOD, (D) GSH and (E) GSH-PX levels. (F) GeneChip analysis. (G) Reverse transcription-quantitative polymerase chain reaction was used to determine miR-214 expression detection. " P<0.01 vs. Sham group. miR, microRNA; DN, diabetic nephropathy; Sham, control group; MDA, malondialdehyde; GSH-PX, glutathione peroxidase; GSH, glutathione; SOD, superoxide dismutase.

using Lipofectamine 2000 (Thermo Fisher Scientific, Inc.). Cells were assayed using luciferase assay kits (Promega Corporation, Madison, WI, USA) for $48 \mathrm{~h}$ after transfection. Renilla luciferase activity was used for normalization.

Statistical analysis. All data were expressed as the mean \pm standard deviation. The Student's t-test or one-way analysis of variance with a Student-Newman-Keuls post hoc test was used to discern individual differences between groups. $\mathrm{P}<0.05$ was considered to indicate a statistically significant difference.

\section{Results}

miR-214 expression in rats with DN. Hematoxylin and eosin staining revealed that the glomerulus was damaged and the volume of the glomerulus was markedly reduced in rats with DN compared with the Sham group (Fig. 1A). Furthermore, there were significantly decreased levels of glutathione peroxidase (GSH-PX), glutathione (GSH) and superoxide dismutase (SOD), but significantly increased levels of malondialdehyde (MDA) in rats with DN compared with the Sham group (Fig. 1B-E). As indicated in Fig. 1F and G, miR-214 expression 

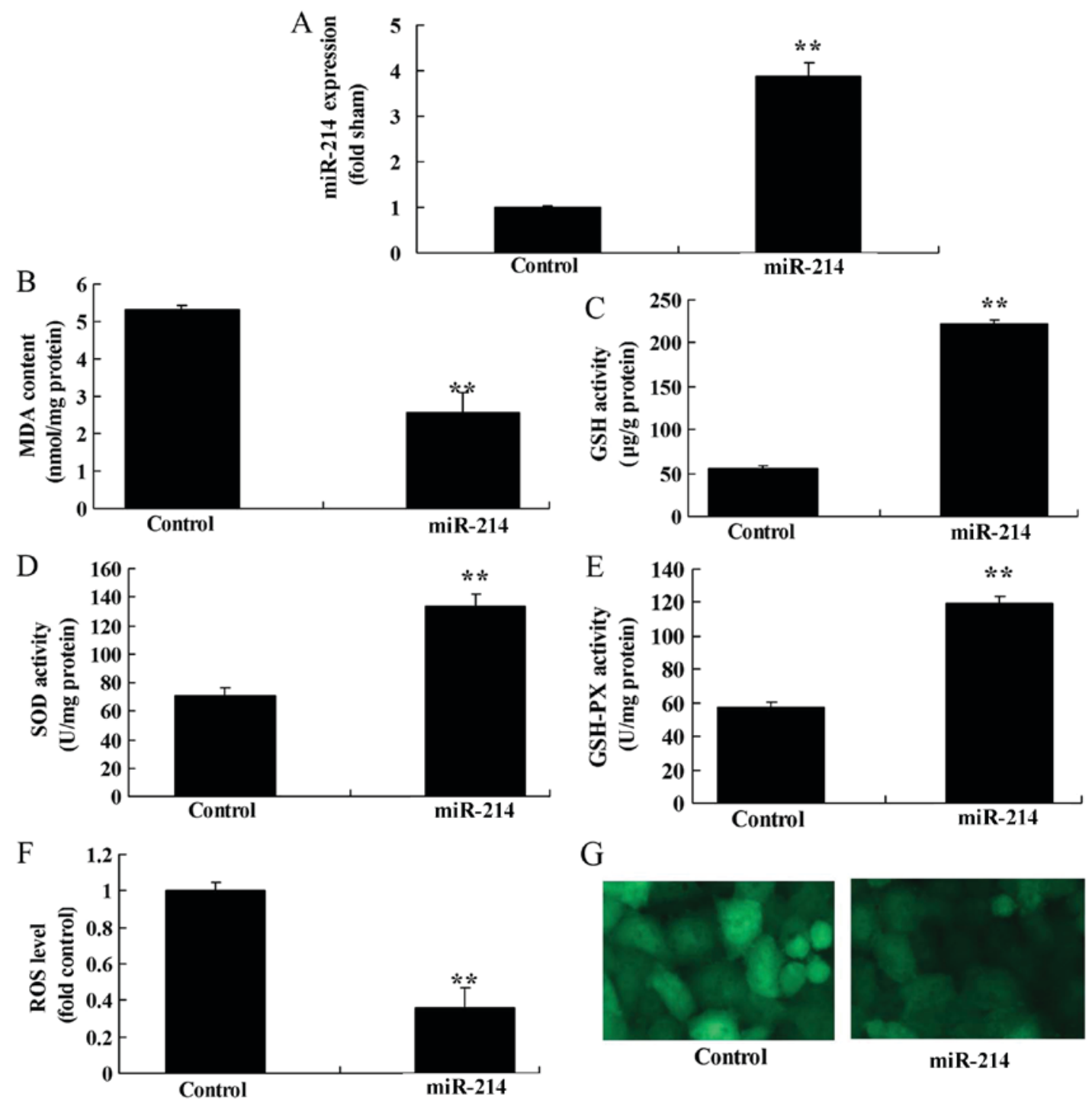

G
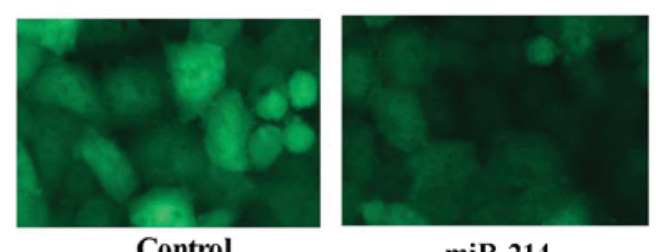

Control

miR-214

Figure 2. Overexpression of miR-214 increases oxidative stress in HK-2 renal proximal tubular epithelial cells. (A) Reverse transcription-quantitative polymerase chain reaction was used to determine miR-214 expression. ELISA was used to determine (B) MDA, (C) SOD, (D) GSH and (E) GSH-PX. (F) ROS levels and (G) ROS probe staining (dichloro-dihydro-fluorescein diacetate; magnification, $\mathrm{x} 200$ ). ${ }^{* *} \mathrm{P}<0.01 \mathrm{vs}$. control group. miR-214, overexpression of miR-214 group; Control, control negative group; miR-214, microRNA-214; MDA, malondialdehyde; GSH-PX, glutathione peroxidase; GSH, glutathione; SOD, superoxide dismutase; ROS, reactive oxygen species.

was significantly decreased in rats with DN compared with the Sham group. These results suggested that miR-214 may regulate the progression of $\mathrm{DN}$.

Overexpression of miR-214 decreases oxidative stress in $\mathrm{HK}-2$ cell. As indicated in Fig. 2, miR-214 overexpression significantly increased the levels of GSH-PX, GSH and SOD, and significantly reduced the levels of MDA in the in vitro model of DN compared with the control group. Furthermore, miR-214 overexpression also significantly reduced ROS levels in DN in vitro when compared with the control group (Fig. $2 \mathrm{E}$ and F). The results suggested that miR-214 could modulate oxidative stress in DN.

Overexpression of miR-214 regulates the Akt/mTOR signaling pathway by UCP2 in HK-2 cell. miR-214 targeted the 3'-untranslated region of UCP2, and luciferase activity was significantly increased in the overexpressed miR-214 group compared with the control group (Fig. 3). As indicated in Fig. 3C-F, miR-214 overexpression significantly increased the protein expression levels of UCP2, p-Akt and p-mTOR in DN in vitro when compared with the control group. These findings indicated that miR-214 regulated the progression of DN through the Akt/mTOR signaling pathway by UCP2.

UCP2 inhibitor attenuates the effects of miR-214 upregulation on oxidative stress in DN in HK-2 cell. The function of UCP2 in DN via miR-214 was assessed in the present study. Results indicated that UCP2 inhibitor, genipin, significantly inhibited UCP2 expression in DN via miR-214 overexpression. As indicated in Fig. 4A-D, UCP2 inhibitor significantly suppressed the protein expression levels of UCP2, p-Akt and p-mTOR in miR-214-upregulated DN cells compared with miR-214 upregulation alone. In addition, UCP2 inhibitor significantly reduced the levels of GSH-PX, GSH and SOD, and significantly increased those of MDA and ROS in miR-214-upregulated DN 
A

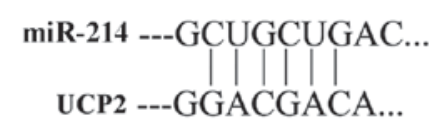
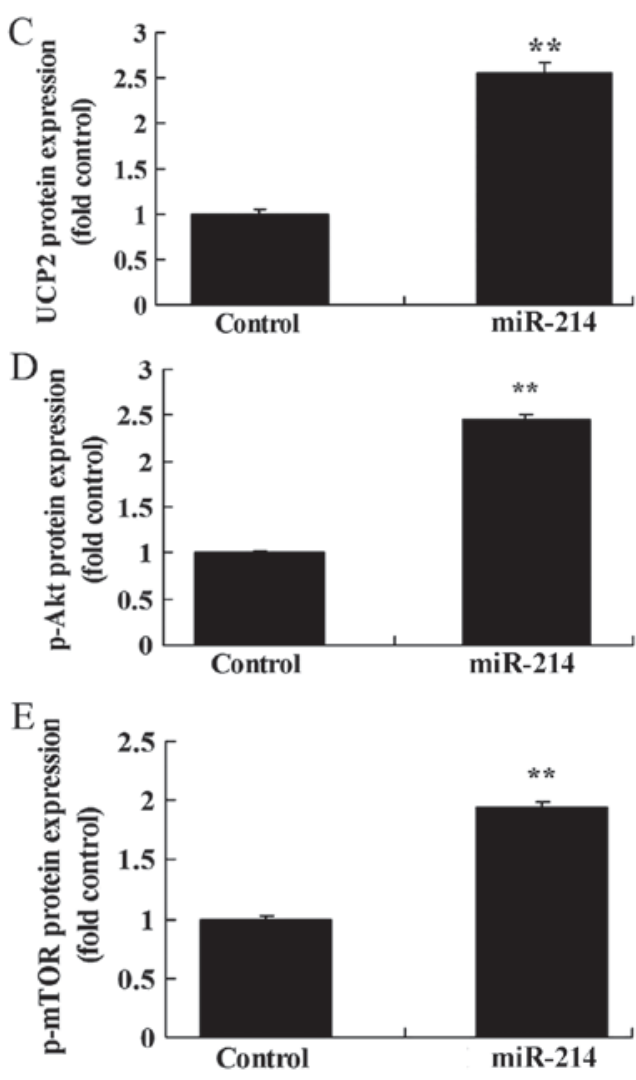
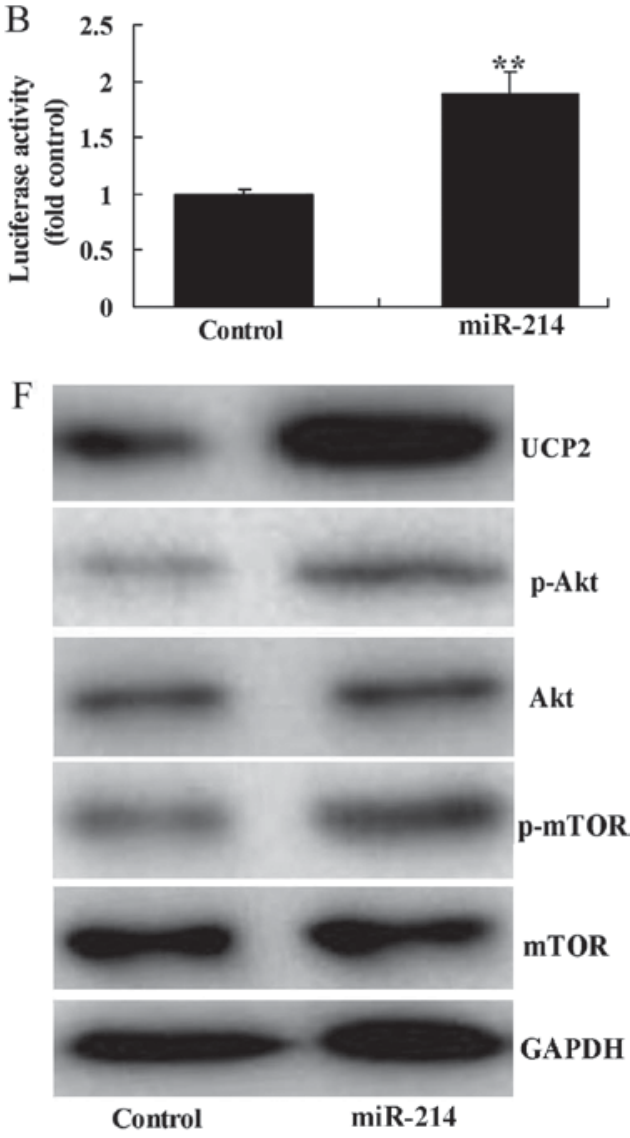

Figure 3. Overexpression of miRNA-214 regulates Akt/mTOR signaling by UCP2. (A) miR-214 targeted the 3'-untranslated region of UCP2. (B) Luciferase activity and (C) UCP2, (D) p-Akt and (E) p-mTOR protein expression levels were determined using statistical analysis. (F) Western blot analysis for UCP2, p-Akt and p-mTOR protein expression. ${ }^{* *} \mathrm{P}<0.01$ vs. control group. miR-214, overexpression of miRA-214 group; Control, control negative group; miR-214, microRNA-214; UCP2, uncoupling protein 2; mTOR, mammalian target of rapamycin; p, phosphorylated.

cells compared with miR-214 upregulation alone (Fig. 4E-J). The results suggested that UCP2 also serves a role in miR-214-regulated oxidative stress in DN.

Akt inhibitor reduces the effects of miR-214 upregulation on oxidative stress in DN in in HK-2 cell. The role of Akt in the function of miR-214 on inflammation in DN was investigated. As indicated in Fig. 5A-C, Akt inhibitor (2 nM MK 2206 dihydrochloride) significantly suppressed the protein expression levels of p-Akt and p-mTOR in miR-214-upregulated DN cells compared with miR-214 upregulation alone. Akt inhibitor significantly reduced the levels of GSH-PX, GSH and SOD, and significantly elevated those of MDA in miR-214-upregulated DN cells compared with miR-214 upregulation alone (Fig. 5D-G). These results suggested that miR-214 regulates the UCP2/ROS/Akt/mTOR signaling pathway to suppress oxidative stress in diabetic nephropathy.

\section{Discussion}

Type 2 diabetes is a common and frequently-occurring endocrine/metabolic disease (13). People's living standards have increasingly improved over time (13). However, unhealthy diets, work pressures, lack of physical exercise and accelerated aging of the population have resulted in increased diabetes-associated morbidity (13). Diabetic retinopathy is the microvascular complication of diabetes, which may result in visual impairment and blindness (3). Thus, the morbidity and rate of blindness of DN are increasing accordingly (3). To the best of our knowledge, the present study is the first to indicate that miR-214 expression levels in peripheral blood were decreased in rats with DN. Notably. Costantino et al (14) revealed that miR-214 is associated with hyperglycemic memory in diabetic mice.

Previous results have indicated that UCP2 can stimulate $B$ cells to secrete insulin (5). Results of experiments on a rat UCP2 model indicated that overexpression of UCP2 led to reduced intracellular ATP content (5). In addition, overexpression of UCP2 decreased the sensitivity of glucose on B cells, which therefore reduced insulin secretion (15). It was also identified in UCP2 gene knockout mice that increased ATP content can be observed in B cells in vivo or in vitro (15). This also increases the sensitivity of glucose to stimulate insulin secretion (15). As demonstrated in previous study, elevated $\mathrm{UCP} 2$ activity or overexpression of UCP2 in B cells may affect insulin secretion $(7,15)$. The correlation of UCP2 gene polymorphisms with diabetes has become a new research focus (7). The current study suggested that miR-214 overexpression 

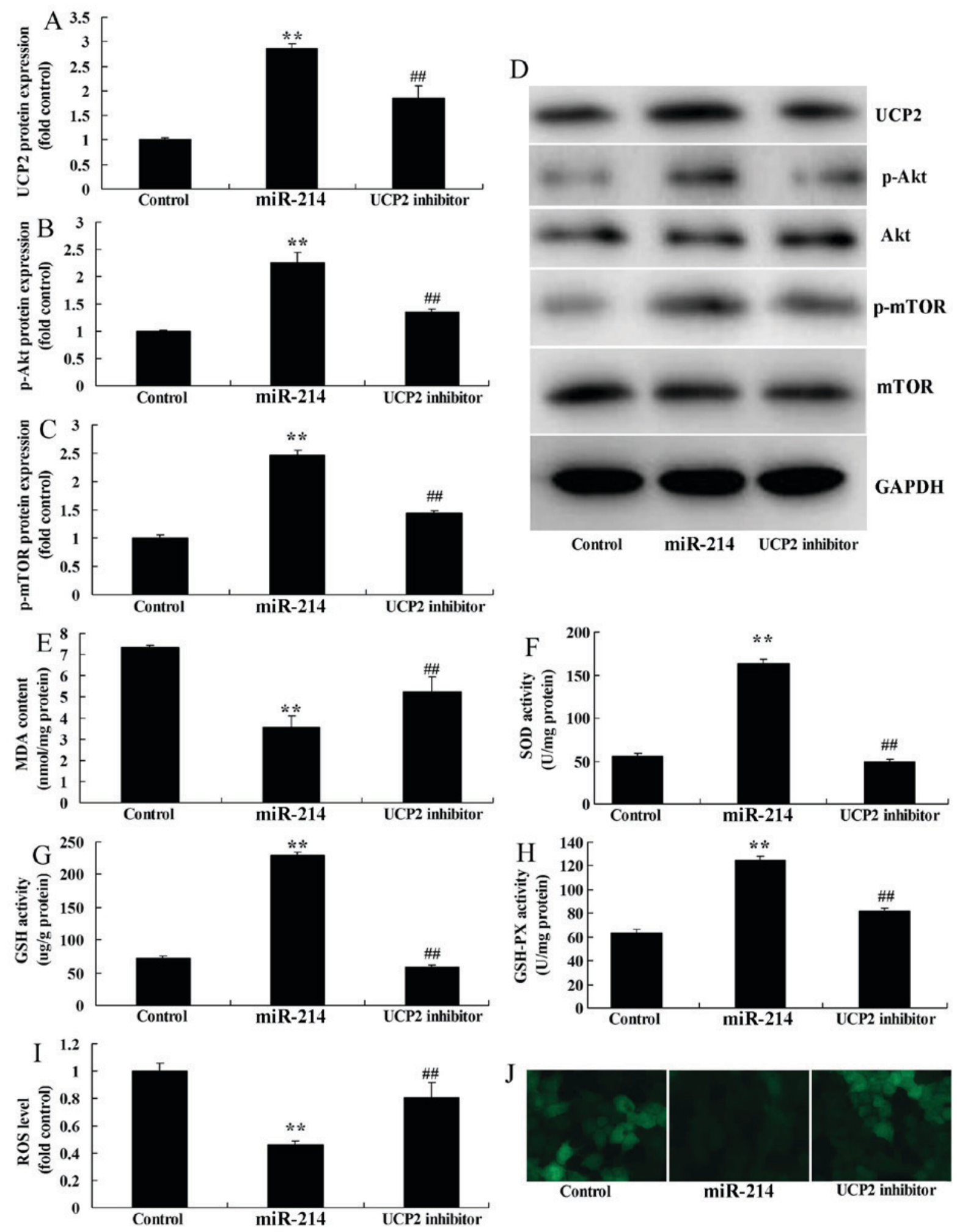

Figure 4. UCP2 inhibitor reduces the effects of miR-214 upregulation on oxidative stress in an in vitro DN model. (A) UCP2, (B) p-Akt and (C) p-mTOR protein expression levels were determined using statistical analysis and (D) western blot analysis. ELISA was used to determine (E) MDA, (F) SOD, (G) GSH, (H) GSH-PX levels and (I) ROS levels and (J) ROS probe staining (dichloro-dihydro-fluorescein diacetate; magnification, $\mathrm{x} 200$ ). ${ }^{* *} \mathrm{P}<0.01$ vs. control group; ${ }^{\# \#} \mathrm{P}<0.01$ vs. miR-214 group. miR-214, overexpression of miR-214 group; Control, control negative group; UCP2 inhibitor, overexpression of miR-214 and UCP2 inhibitor group; miR-214, microRNA-214; UCP2, uncoupling protein 2; mTOR, mammalian target of rapamycin; p, phosphorylated; MDA, malondialdehyde; GSH-PX, glutathione peroxidase; GSH, glutathione; SOD, superoxide dismutase; ROS, reactive oxygen species.

induced UCP2 protein expression in the in vitro DN model. Furthermore, Yu et al (16) indicated that miR-214 is a putative tumor suppressor by targeting UCP2 in hepatocellular carcinoma.
Oxidative stress markers are a series of biochemical substances that reflect oxidative stress levels in vivo (17). There are various types of oxidative stress markers. Of them, ROS, reactive nitrogen species and lipid peroxide are commonly used 

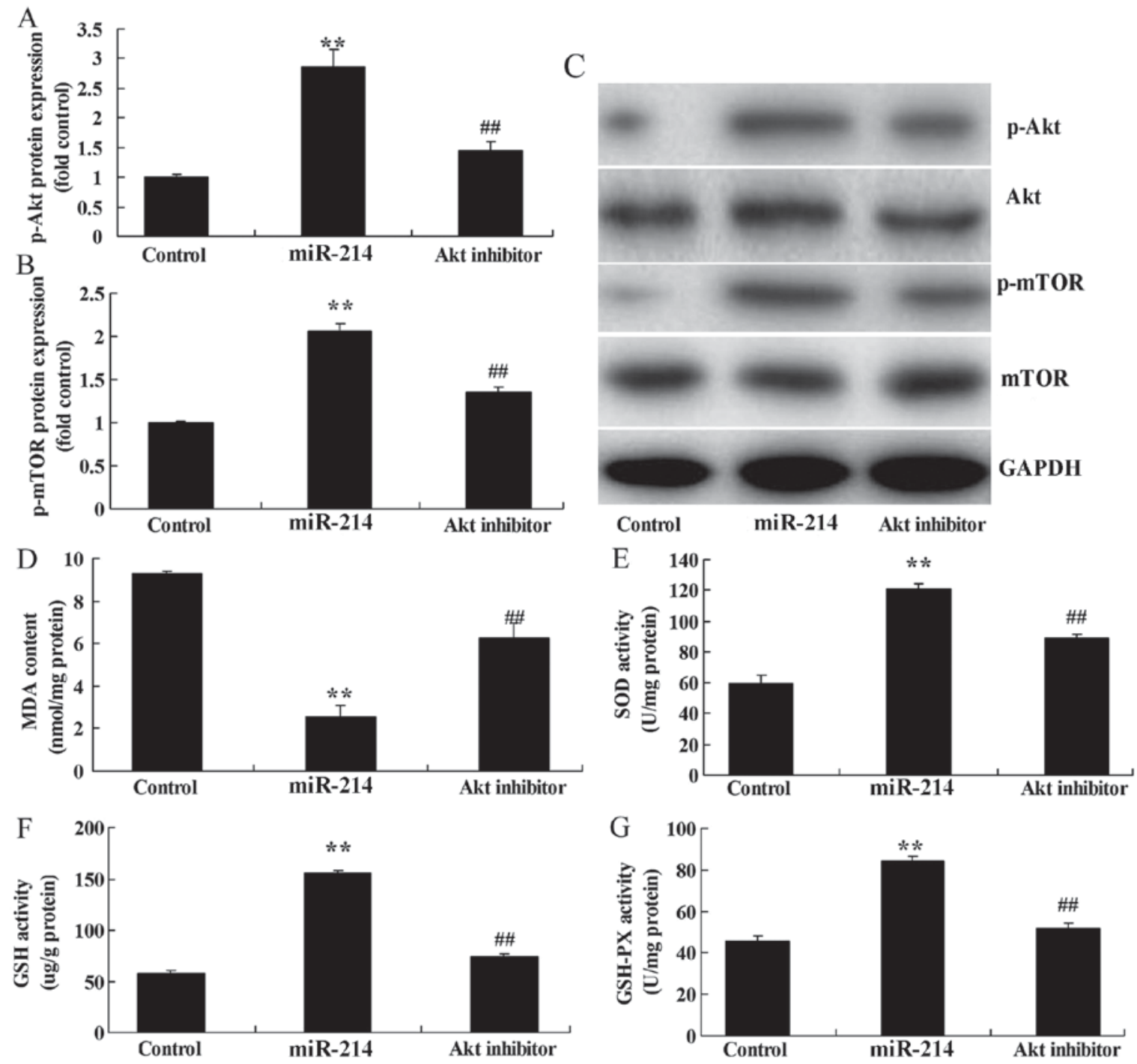

Figure 5. Akt inhibitor reduces the effects of miR-214 upregulation on oxidative stress in an in vitro DN model. (A) p-Akt and (B) p-mTOR protein expression using statistical analysis. (C) Western blot analysis for p-Akt and p-mTOR protein expression. ELISA was used to determine (D) MDA, (E) SOD, (F) GSH and (G) GSH-PX. ${ }^{* *} \mathrm{P}<0.01$ vs. control group; ${ }^{\# \#} \mathrm{P}<0.01$ vs. miR-214 group. miR-214, overexpression of miR-214 group; Control, control negative group; Akt inhibitor, overexpression of miR-214 and Akt inhibitor group; miR-214, microRNA-214; mTOR, mammalian target of rapamycin; p, phosphorylated; MDA, malondialdehyde; GSH-PX, glutathione peroxidase; GSH, glutathione; SOD, superoxide dismutase.

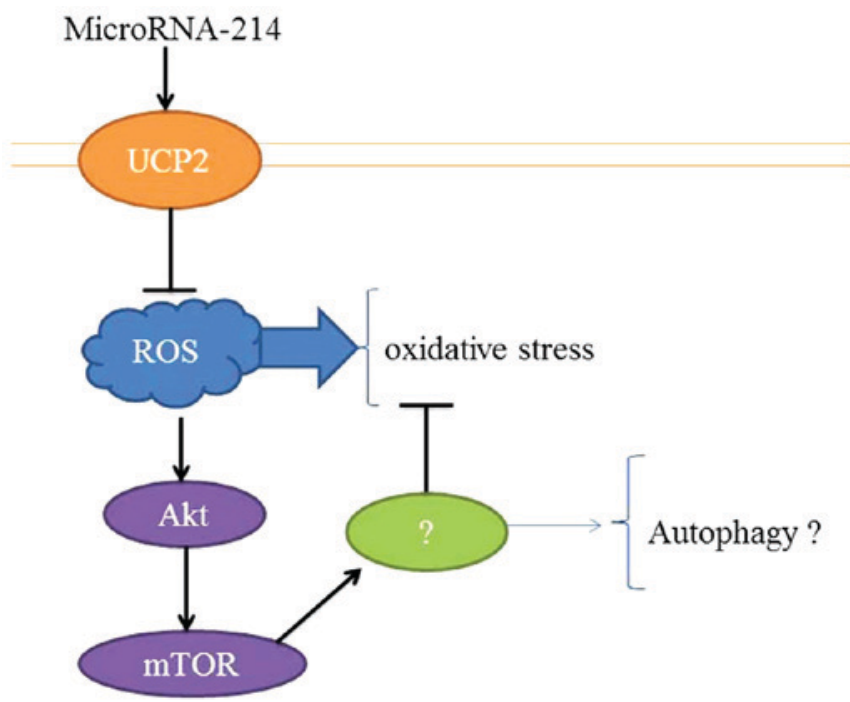

Figure 6. MicroRNA-214 suppresses oxidative stress in diabetic nephropathy via the ROS/Akt/mTOR signaling pathway by UCP2. ROS, reactive oxygen species; mTOR, mammalian target of rapamycin; UCP2, uncoupling protein 2. 
in diabetes detection (17). Determination of oxidative stress markers serves an important role in revealing correlations of diabetes genesis and development with oxidative stress (17). In the present study, it was observed that miR-214 overexpression decreased oxidative stress in the in vitro DN model. Notably, Gao et al (18) revealed that miR-214 protects erythroid cells against oxidative stress by targeting activating transcription factor 4 and enhancer of zeste homolog 2 (EZH2). These results demonstrated that miR-214 overexpression decreased oxidative stress in DN.

The inflammation-associated p38 mitogen-activated protein kinase (MAPK) signaling pathway is associated with renal tissue injury during the development of DN (19). Multiple factors, including hyperglycemia, abnormal hemodynamics, oxidative stress and pre-inflammatory factors, can activate the downstream p38 MAPK signaling pathway (20). The activated p38 MAPK signal pathway can further induce activation of downstream inflammatory cells. Furthermore, it promotes the expression of inflammatory mediators, and increases the production of inflammatory factors (20). Finally, the p38 MAPK signaling pathway leads to inflammatory injury of renal tissues. The intervention of the p38 MAPK signaling pathway is achieved through multiple approaches.

Akt is the downstream signaling molecule of phosphatidylinositol-4,5-bisphosphate 3-kinase (PI3K) (21). Notably, the $\mathrm{PI} 3 \mathrm{~K} / \mathrm{Akt}$ signaling pathway serves an important role in insulin signal transmission (22). Such signaling systems have an important role in biological processes (22). These include metabolism, cell growth, proliferation, survival and migration, as well as cytoskeletal reconstruction, inflammation, cell apoptosis, membrane transport and secretion (23). Furthermore, the PI3K/Akt signaling pathway can promote cell survival, differentiation and growth (23). Studies have indicated that the PI3K/Akt signaling pathway is associated with vascular smooth muscle contraction, endothelium-mediated relaxation and blood pressure (24). The PI3K/Akt signaling pathway is also extensively involved in the formation of atherosclerosis, pathological angiogenesis, inflammatory cell aggregation and vascular smooth muscle cell dysfunction (23). Additionally, the PI3K/Akt signaling pathway can promote vasoconstriction, induce vascular remodeling and accelerate the genesis and development of atherosclerosis (25). It was identified that miR-214 overexpression induced the Akt/mTOR signaling pathway in an in vitro DN model in the present study. Das et al (26) revealed that miR-214 reduces insulin like growth factor 1 receptor expression and downstream mTOR complex 1 signaling in renal carcinoma cells. These results demonstrated that $\mathrm{miR}-214$ suppressed oxidative stress in DN via UCP2 in the ROS/Akt/mTOR signaling pathway.

Fig. 6 revealed a schematic diagram of the suggested potential mechanism involved in the function of miR-214 on oxidative stress in DN. In conclusion, the present study demonstrated for the first time that miR-214 may regulate diabetes through the insulin and ROS/Akt/mTOR signaling pathways in proximal tubular cells by UCP2. Collectively, these results suggest that miR-214 has protective effects against oxidative stress in DN, which could be used in clinical applications.

\section{Acknowledgements}

Not applicable.

\section{Funding}

No funding was received.

\section{Availability of data and materials}

The datasets used and/or analyzed during the current study are available from the corresponding author on reasonable request.

\section{Authors' contributions}

SY designed the experiment. XF, YL, BX, YM and HW performed the experiments. SY analyzed the data and prepared the manuscript. All authors read and approved the final version of the manuscript.

\section{Ethics approval and consent to participate}

The present study was approved by the Taizhou People's Hospital Ethics Committee (Taizhou, China).

\section{Patient consent for publication}

Not applicable.

\section{Competing interests}

The authors declare that they have no competing interests.

\section{References}

1. Lindhardt M, Persson F, Currie G, Pontillo C, Beige J, Delles C, von der Leyen H, Mischak H, Navis G, Noutsou M, et al: Proteomic prediction and Renin angiotensin aldosterone system Inhibition prevention Of early diabetic nephRopathy in TYpe 2 diabetic patients with normoalbuminuria (PRIORITY): Essential study design and rationale of a randomised clinical multicentre trial. BMJ Open 6: e010310, 2016.

2. Nakamura T, Sato E, Amaha M, Kawagoe Y, Maeda S and Yamagishi S: Addition of aliskiren to angiotensin II receptor blockers ameliorates renal tubular injury and reduces intima media thickness of carotid artery in patients with diabetic nephropathy. Int J Cardiol 155: 294-296, 2012.

3. Koya D, Haneda M, Inomata S, Suzuki Y, Suzuki D, Makino H, Shikata K, Murakami Y, Tomino Y, Yamada K, et al: Long-term effect of modification of dietary protein intake on the progression of diabetic nephropathy: A randomised controlled trial. Diabetologia 52: 2037-2045, 2009.

4. Abe M, Higuchi T, Moriuchi M, Okamura M, Tei R, Nagura C, Takashima H, Kikuchi F, Tomita H and Okada K: Efficacy and safety of saxagliptin, a dipeptidyl peptidase-4 inhibitor, in hemodialysis patients with diabetic nephropathy: A randomized open-label prospective trial. Diabetes Res Clin Pract 116: 244-252, 2016.

5. Friederich-Persson M, Aslam S, Nordquist L, Welch WJ, Wilcox CS and Palm F: Acute knockdown of uncoupling protein-2 increases uncoupling via the adenine nucleotide transporter and decreases oxidative stress in diabetic kidneys. PLoS One 7: e39635, 2012.

6. Qiu W, Zhou Y, Jiang L, Fang L, Chen L, Su W, Tan R, Zhang CY, Han X and Yang J: Genipin inhibits mitochondrial uncoupling protein 2 expression and ameliorates podocyte injury in diabetic mice. PLoS One 7: e41391, 2012.

7. de Souza BM, Michels M, Sortica DA, Bouças AP, Rheinheimer J, Buffon MP, Bauer AC, Canani LH and Crispim D: Polymorphisms of the UCP2 gene are associated with glomerular filtration rate in type 2 diabetic patients and with decreased UCP2 gene expression in human kidney. PLoS One 10: e0132938, 2015.

8. Nassirpour R, Raj D, Townsend R and Argyropoulos C: MicroRNA biomarkers in clinical renal disease: From diabetic nephropathy renal transplantation and beyond. Food Chem Toxicol 98: 73-88, 2016 
9. Lee SY and Choi ME: Urinary biomarkers for early diabetic nephropathy: Beyond albuminuria. Pediatr Nephrol 30: 1063-1075, 2015.

10. von Brandenstein M, Pandarakalam JJ, Kroon L, Loeser H, Herden J, Braun G, Wendland K, Dienes HP, Engelmann U and Fries JW: MicroRNA 15a, inversely correlated to PKCalpha, is a potential marker to differentiate between benign and malignant renal tumors in biopsy and urine samples. Am J Pathol 180: 1787-1797, 2012

11. Yamada H, Itoh M, Hiratsuka I and Hashimoto S: Circulating microRNAs in autoimmune thyroid diseases. Clin Endocrinol (Oxf) 81: 276-281, 2014.

12. Livak KJ and Schmittgen TD: Analysis of relative gene expression data using real-time quantitative PCR and the 2(-Delta Delta C(T)) method. Methods 25: 402-408, 2001.

13. Araki H, Kuwagata S, Soumura M, Yamahara K, Morita Y, Kume S, Isshiki K, Araki S, Kashiwagi A, Maegawa $\mathrm{H}$ and Uzu T: Safety and efficacy of skin patches containing loxoprofen sodium in diabetic patients with overt nephropathy. Clin Exp Nephrol 18: 487-491, 2014.

14. Costantino S, Paneni F, Lüscher TF and Cosentino F: MicroRNA profiling unveils hyperglycaemic memory in the diabetic heart. Eur Heart J 37: 572-576, 2016.

15. Persson MF, Franzén S, Catrina SB, Dallner G, Hansell P, Brismar K and Palm F: Coenzyme Q10 prevents GDP-sensitive mitochondrial uncoupling, glomerular hyperfiltration and proteinuria in kidneys from $\mathrm{db} / \mathrm{db}$ mice as a model of type 2 diabetes. Diabetologia 55: 1535-1543, 2012.

16. Yu G, Wang J, Xu K and Dong J: Dynamic regulation of uncoupling protein 2 expression by microRNA-214 in hepatocellular carcinoma. Biosci Rep 36: e00335, 2016.

17. Wang X, Huang R, Zhang L, Li S, Luo J, Gu Y, Chen Z, Zheng Q, Chao T, Zheng W, et al: A severe atherosclerosis mouse model on the resistant NOD background. Dis Model Mech 11: dmm033852, 2018.

18. Gao M, Liu Y, Chen Y, Yin C, Chen JJ and Liu S: miR-214 protects erythroid cells against oxidative stress by targeting ATF4 and EZH2 Free Radic Biol Med 92: 39-49, 2016.

19. Wang T, Chen SS, Chen R, Yu DM and Yu P: Reduced beta 2 glycoprotein I improve diabetic nephropathy via inhibiting TGF-ß1-p38 MAPK pathway. Int J Clin Exp Med 8: 6852-6865, 2015.
20. Lu HJ, Tzeng TF, Liou SS, Da Lin S, Wu MC and Liu IM: Polysaccharides from Liriopes Radix ameliorate streptozotocin-induced type I diabetic nephropathy via regulating $\mathrm{NF}-\kappa \mathrm{B}$ and $\mathrm{p} 38 \mathrm{MAPK}$ signaling pathways. BMC Complement Altern Med 14: 156, 2014.

21. Sun L, Li W, Li W, Xiong L, Li G and Ma R: Astragaloside IV prevents damage to human mesangial cells through the inhibition of the NADPH oxidase/ROS/Akt/NF- $\kappa \mathrm{B}$ pathway under high glucose conditions. Int J Mol Med 34: 167-176, 2014.

22. Xu F, Wang Y, Cui W, Yuan H, Sun J, Wu M, Guo Q, Kong L, $\mathrm{Wu} \mathrm{H}$ and Miao L: Resveratrol prevention of diabetic nephropathy is associated with the suppression of renal inflammation and mesangial cell proliferation: Possible roles of Akt $/ \mathrm{NF}-\kappa \mathrm{B}$ pathway. Int J Endocrinol 2014: 289327, 2014.

23. Song Z, Guo Y,Zhou M and Zhang X: The PI3K/p-Akt signaling pathway participates in calcitriol ameliorating podocyte injury in DN rats. Metabolism 63: 1324-1333, 2014.

24. Huang G, Lv J, Li T, Huai G, Li X, Xiang S, Wang L, Qin Z, Pang J, Zou B and Wang Y: Notoginsenoside R1 ameliorates podocyte injury in rats with diabetic nephropathy by activating the PI3K/Akt signaling pathway. Int J Mol Med 38: 1179-1189, 2016.

25. Ji X, Li C, Ou Y, Li N, Yuan K, Yang G, Chen X, Yang Z, Liu B, Cheung WW, et al: Andrographolide ameliorates diabetic nephropathy by attenuating hyperglycemia-mediated renal oxidative stress and inflammation via Akt/NF- $\mathrm{kB}$ pathway. Mol Cell Endocrinol 437: 268-279, 2016.

26. Das F, Dey N, Bera A, Kasinath BS, Ghosh-Choudhury N and Choudhury GG: MicroRNA-214 reduces insulin-like growth factor-1 (IGF-1) receptor expression and downstream mTORC1 signaling in renal carcinoma cells. J Biol Chem 291: 14662-14676, 2016.

This work is licensed under a Creative Commons Attribution-NonCommercial-NoDerivatives 4.0 International (CC BY-NC-ND 4.0) License. 